Intemational Journal of Modern Physics B, Vol. 7, Nos. 6 \& 7 (1993) 1505-1525

(c) World Scientific Publishing Company

\title{
THE PHONON SPECTRUM OF THE OCTAGONAL TILING
}

\author{
J. LOS, T. JANSSEN \\ Institute for Theoretical Physics, University of Nijmegen, 6525 ED Nijmegen, \\ The Netherlands \\ and \\ F. GÄHLER \\ Département de Physique Theorique, Université de Genève, \\ 24 quai Ernest Ansermet, CH-1211 Genève, Switzerland
}

\begin{abstract}
A study of the phonon spectrum of the octagonal tiling is presented, by calculating and analysing the properties of the spectrum of perfect and randomized commensurate approximants with unit cells containing up to 8119 vertices. The total density of states, obtained by numerical integration over the Brillouin zone, exhibits much structure, and in the low frequency range of the spectrum there is deviation from the normal linear behaviour in the form of pseudogaps. For randomized approximants these pseudogaps disappear and the density of states is globally smoothened. It turns out that the widths of the gaps in the dispersion vanish in the low frequency limit. Therefore the scaling behaviour of the lowest branches tends to the behaviour of an absolutely continuous spectrum, which is not the case at higher frequencies. As an application, the vibrational specific heat of the different tiling models is calculated and compared to the specific heat of a square lattice and of a Debye model.
\end{abstract}

\section{Introduction}

The octagonal tiling is a model for a 2-dimensional quasicrystal. It has no lattice periodicity, but is quasiperiodic. For this reason the calculation of physical properties, such as phonons and electronic structure, is more difficult than for ordinary crystals. Most of the research concerning the physical properties of quasicrystals has been devoted to one-dimensional models, such as the Fibonacci chain. For the Fibonacci chain and similar systems it has been shown that their electron or phonon spectrum shows scaling behaviour. ${ }^{1}$ The spectrum of the Fibonacci chain is neither absolutely continuous nor a pure point spectrum, but singularly continuous. Correspondingly the eigenstates are neither extended nor localized, but critical. ${ }^{2}$ In the present paper, concerning the dynamics of a 2-dimensional quasicrystal, we will restrict ourselves to the properties of the spectrum, in particular the fine structure of the density of states (DOS) and the scaling behaviour of the phonon branches for commensurate approximants. A study of the eigenvectors will be presented elsewhere. 
In three dimensions calculations have been done on the phonon spectrum of clusters and commensurate approximants of the icosahedral Penrose tiling, ${ }^{3,4,5}$ and on clusters of Danzer's icosahedral tiling. ${ }^{6,7}$ Both tilings are used as models for icosahedral quasicrystals. For a simple dynamical model with atoms on the vertices of the tiling and springs between neighbouring atoms, the density of states (DOS) turned out to be globally rather structureless. ${ }^{3}$ A more recent study $y^{4,5}$ on the effect of random tiling type disorder (see Sec. 2), which might be present in real quasicrystals, demonstrated that the DOS is even smoother for randomized approximants. However, in all these calculations only a limited number of states was considered, which might be insufficient to conclude on the fine structure of the spectrum and the DOS in the quasiperiodic limit. For 2-dimensional systems, however, it should be much easier to study systems large enough to be able to conclude on the infinite volume behaviour. Among the 2-dimensional systems that have been studied so far are the Penrose tiling ${ }^{8}$ and, most recently, the octagonal tiling. ${ }^{9}$ The methods used in Ref. 9 are different from ours, however.

For a periodic structure, the DOS exhibits a number of singularities, so-called van Hove singularities, which are due to stationary points in the phonon branches, where $\nabla_{k} \omega_{d}(k)=0$. For a quasiperiodic structure there is, a priori, no band structure at all, and it is not clear what should be expected. A quasi-periodic structure can, however, be viewed as the limit of a sequence of commensurate approximants, and for these approximants the spectrum will certainly contain van Hove singularities. These singularities might become weaker, however, when tending to the quasiperiodic limit, since the size of the Brillouin zone becomes small for large approximants.

In the present paper we present a Brillouin zone integration method to obtain the DOS, which is applied to a sequence of successive commensurate approximants of the octagonal tiling (OT-approximants). From these results we can then extrapolate to the behaviour of the DOS in the incommensurate limit. We also study how the details of the DOS are affected by randomization.

Our Brillouin zone integration method is in principle based on local quadratic interpolation of the branch surfaces. Such a quadratic fit was also used by MacDonald $e t$ al. ${ }^{10}$ and by Methfessel ${ }^{11}$ for three-dimensional systems, but can be used in two dimensions as well. In our method we follow the so-called hybrid method of MacDonald and introduce a further refinement in order to be able to trace as well as possible the details of the DOS, in particular the singularities. Our method is, moreover, very efficient.

Special attention will be paid to the low frequency part of the spectrum, in an attempt to answer the question whether there is deviation from the normal linear behaviour of the DOS. For periodic two-dimensional structures the linear behaviour of the DOS at low frequencies is a direct consequence of the linear dispersion of the accoustic branches near $k=0$. For the Fibonacci chain it has been shown, by considering successive rational approximants, that the width of the gaps relative to 
the bandwidths tends to zero in the low frequency limit. ${ }^{12}$ For the DOS this means that in the Fibonacci case one may expect a normal constant behaviour at low frequencies, with eventually small deviations occurring within very small intervals in w-space.

For one dimensional quasi-periodic systems the character of the spectrum can be determined by analysing the decay of the bandwidths for a systematic sequence of commensurate approximants. In principle, this approach can be generalized to two- and three-dimensional systems. In Ref. 13 the scaling behaviour of the bandwidths was represented by an 'entropy' function $S(\epsilon)$, which is calculated using a thermodynamical formalism. In the present paper we propose a slightly different method, without using the thermodynamical formalism. Instead of considering the spectral $S(\epsilon)$-function, we focus on a function which is more directly related to the distribution of scaling indices $\Omega(\epsilon)$, in order to display more of the details of this distribution. In the thermodynamical formalism these details would remain hidden.

The remainder of the paper is organised as follows. In Sec. 2 the different model structures are presented. The octagonal tiling and its commensurate approximants are described, and randomized approximants are introduced. The dynamical model based on these tilings then is defined. In Sec. 3 the Brillouin zone integration method is described, and the DOS is presented for various perfect and randomized approximants. As an application, the specific heat is calculated for the different models, and it is compared to the specific heat of a corresponding square lattice model, and to the specific heat of a Debye model. The low frequency range of the spectrum then is considered in more detail. In Sec. 4, the scaling behaviour of the phonon branches is discussed, before we conclude in Sec. 5 .

\section{The Model Structures}

\subsection{The octagonal tiling and its commensurate approximants}

Octagonal tilings, discovered independently by R. Ammann (see Ref. 14) and $F$. Beenker, ${ }^{15}$ are quasiperiodic coverings of the plane by squares and $45^{\circ}$ rhombuses. They are eightfold symmetric in the sense that the rotated tiling is locally indistinguishable from the original tiling. Their Fourier transform consists of a rank 4 module of Bragg peaks, which is eightfold symmetric and has an eightfold symmetric intensity distribution. Octagonal tilings can be obtained from a 4-dimensional hypercubic lattice by the cut and projection method. ${ }^{16}$ Let $V_{E} \oplus V_{I}$ be the decomposition of 4-dimensional space into external space $V_{E}$ (also called physical space), and its orthogonal complement $V_{I}$ (internal space), such that the standard basis $e_{1}, \ldots, e_{4}$ of the 4-dimensional hypercubic lattice, with lattice constant $\sqrt{2}$, decomposes into the vectors

$$
\begin{aligned}
& \mathbf{e}_{1}^{E}=(1,0), \mathbf{e}_{2}^{E}=(1 / \sqrt{2}, 1 / \sqrt{2}), \quad \mathbf{e}_{3}^{E}=(0,1), \quad \mathbf{e}_{3}^{E}=(-1 / \sqrt{2}, 1 / \sqrt{2}), \\
& \mathrm{e}_{1}^{I}=(1,0), \quad \mathrm{e}_{2}^{I}=(-1 / \sqrt{2}, 1 / \sqrt{2}), \quad \mathrm{e}_{3}^{I}=(0,-1), \quad \mathrm{e}_{3}^{I}=(1 / \sqrt{2}, 1 / \sqrt{2}),
\end{aligned}
$$


which all are expressed in a suitable orthonormal basis of $V_{E}$ or $V_{I}$, respectively. The vertices of an octagonal tiling are now given by the external components $x_{E}$ of all those lattice points $x=x_{E}+x_{I}$ whose internal component $x_{I}$ is contained in a set $W \subset V_{I}$, called acceptance domain, which is a translate of the projection of a primitive hypercubic unit cell of the lattice onto $V_{I}$. Different translates of $W$ usually give rise to different octagonal tilings.

Octagonal tilings are non-periodic by construction. It is possible, however, to construct a sequence of periodic approximants to an octagonal tiling, with larger and larger unit cells, which converges to an octagonal tiling. For that purpose, the internal components $\mathbf{e}_{i}^{I}$ of the basis vectors of the hypercubic lattice are slightly deformed, so that any two of these internal components become commensurate with the other two. In this case there are then nontrivial lattice vectors with vanishing internal component, i.e. they are entirely contained in external space. Such deformations are obtained if we replace $\sqrt{2}$ by one of its continued fraction approximants $p / q$ in the second line of (1). Provided we use as acceptance domain $W$ a translate of the projection of the correspondingly deformed hypercubic unit cell, the resulting structure then is again a square-rhombus tiling and is lattice periodic. The lattice of translation symmetries is a square lattice with lattice constant

$p+q \sqrt{2}$. If $p / q$ converges to $\sqrt{2}$, the corresponding approximant converges to an octagonal tiling. The lowest such approximants are 1/1-, 3/2-, 7/5-, 17/12- and 41/29-approximants, with respectively $7,41,239,1393$ and 8119 vertices in the unit cell. We usually label the approximants with the number of vertices contained in the unit cell, e.g. 7-approximant, 41-approximant, etc. As an example, a unit cell of the 239-approximant is shown in Fig. 1a. We note that all these approximants have as the only exact symmetry element one mirror line along one of the diagonals of their unit cell. Between two successive approximants, the number of vertices increases approximately by $(1+\sqrt{2})^{2}$.

\subsection{Randomized Approximants}

In addition to the approximants described in the preceding section, which we call perfect approximants, because they approximate, given the lattice periodicity, as perfectly as possible an octagonal tiling, we also consider random tiling approximants. Random tilings are members of a statistical ensemble of tilings. This ensemble contains all possible square-rhombus tilings covering the whole plane, and all such tilings have the same statistical weight. A typical representative in this ensemble resembles, however, a quasiperiodic octagonal tiling, wherefore random tilings may serve as a model for quasicrystals. For further details on random tilings, we refer to Refs. 17, 18.

Here, we consider only the subensemble of random tilings with a given lattice periodicity. Representatives of such periodic random tilings can be produced by a Monte Carlo algorithm, by successively disordering a perfect approximant. For this reason, we call these periodic random tilings randomized approximants. In 


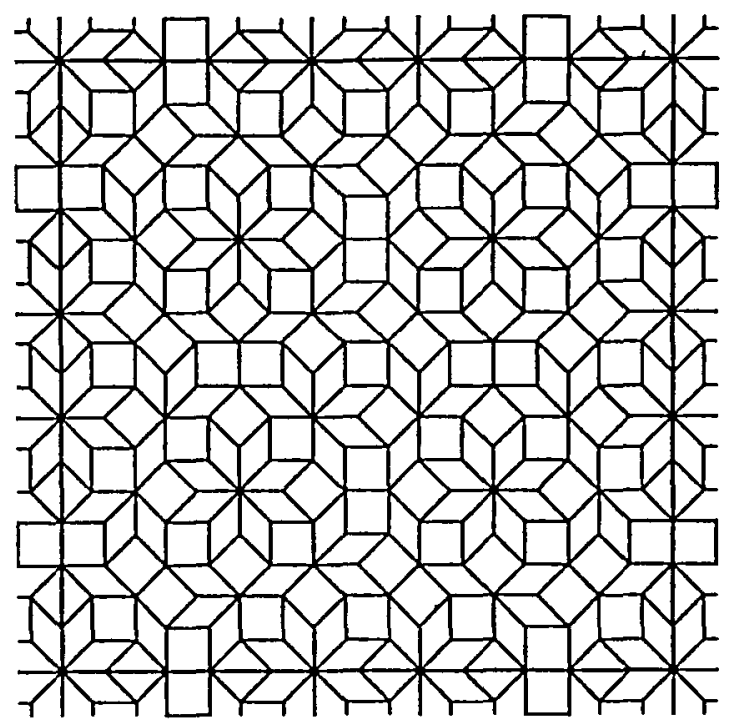

Fig. 1a. The unit cell of a perfect 239-approximant.

each step of this Monte Carlo algorithm, a site is chosen at random. If this site touches only three tiles, a square and two rhombuses, then the hexagon formed by these three tiles is replaced by its mirror image, i.e. the three tiles are reordered. This step is repeated again and again, resulting, after a large number of steps, in a completely randomized approximant. It can be shown that each Monte Carlo step satisfies detailed balance, and that, by the elementary steps as described above, every square-rhombus tiling with the same periodicity can be obtained. Therefore, this Monte Carlo algorithm is ergodic in the space of all periodic square-rhombus tilings and effectively simulates the statistical ensemble of periodic random tilings. An example of a randomized 239-approximant is shown in Fig. 1 b.

\subsection{The Dynamical Model}

Given a periodic square-rhombus tiling as described above, the dynamical model is constructed as follows. On each vertex, an atom of unit mass is placed, and pairs of atoms with a distance not bigger than $\sqrt{2}$ are connected by springs. In other words, there are springs along the short diagonals of the rhombuses, along the tile edges, and along the square diagonals. For simplicity, we have chosen all springs to have the same strength. Different spring constants for different kinds of bonds are not expected to change the qualitative properties of the spectrum (see also Refs. 3, $4,5)$. For each approximant the spring constant $\alpha$ was chosen such that $\gamma \alpha=8$, where $\gamma$ is the mean number of neighbours per atom. In this way we obtain models which may be compared quantitatively with a square lattice, where each atom is 


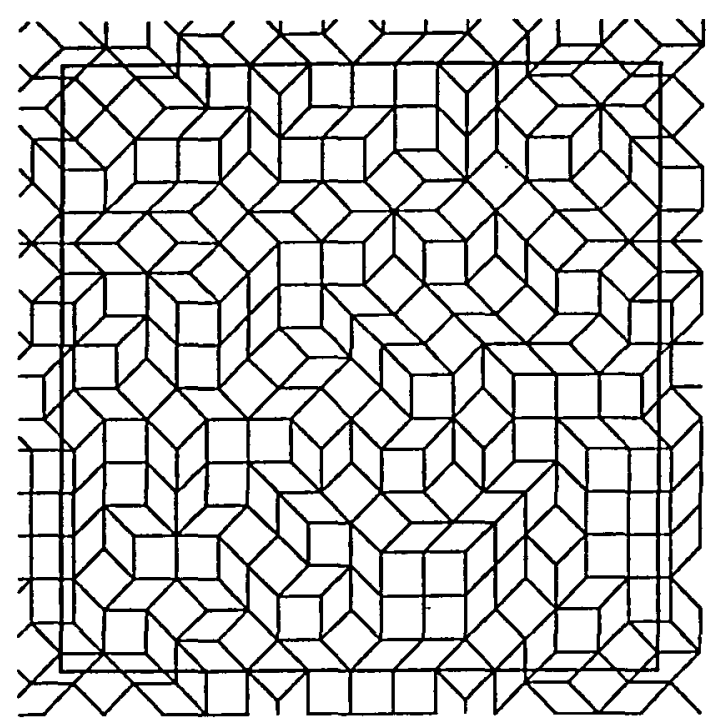

Fig. 1b. The unit cell of a randomized 239-approximant.

connected to its eight neighbours by a spring with spring constant equal to 1 . The potential energy of this system of vibrating masses and springs is given by:

$$
\Phi=\frac{\alpha}{4} \sum_{i} \sum_{i^{\prime}(i)}\left[\left|\mathbf{r}_{i}+\mathbf{u}_{i}-\mathbf{r}_{i^{\prime}}-\mathbf{u}_{i^{\prime}}\right|-\left|\mathbf{r}_{i}-\mathbf{r}_{i^{\prime}}\right|\right]^{2}
$$

where the summation $\sum_{i^{\prime}(i)}$ runs over all neighbours of $i$ and $u_{i}$ is the displacement of atom $i$ relative to its equilibrium position $\mathbf{r}_{i}$. The potential energy (2) is then evaluated in harmonic approximation, i.e. it is replaced by a quadratic form in the displacements $\mathbf{u}_{i}$ of the atoms, by truncating higher order terms in these displacements. Using Born-von Karman boundary conditions leads to a finite dimensional $\mathbf{k}$-dependent dynamical matrix, where $\mathbf{k}$ is a wave vector inside the Brillouin zone. The eigenvalues of the dynamical matrix are the squared eigenfrequencies for the eigenmodes with wavevector $\mathbf{k}$, and can be determined by numerical diagonalisation.

\section{The Fine Structure of the Density of States}

Since the OT-approximants are periodic, normal lattice dynamics can be applied. To obtain the details of the total density of states (DOS) for a periodic structure, it is often not enough to compute the eigenfrequencies for only a finite number of states and then construct from these a histogram. Instead one has to consider all states, by integrating over all wave vectors inside the Brillouin zone. 


\subsection{The Brillouin zone integration method}

For a $d$-dimensional periodic structure the density of states is given by:

$$
D(\omega)=\frac{1}{(2 \pi)^{d}} \sum_{,} \int_{B Z} d \mathbf{k} \delta\left(\omega-\omega_{3}(\mathbf{k})\right)
$$

where $\sum_{s}$ means summation over all branches. If $\hat{\mathbf{n}}$ denotes a unit vector orthogonal to the branch surface at wave vector $\mathbf{k}$, this expression is equivalent to:

$$
D(\omega)=\frac{1}{(2 \pi)^{d}} \sum_{s S} \frac{1}{\left|\hat{\mathbf{n}} \nabla \omega_{s}(\mathbf{k})\right|},
$$

where $\int_{d S}$ means integration over the surface of constant frequency, which in two dimensions is a 1-dimensional curve. For the approximants of the octagonal tiling considered in this paper, the Brillouin zone is a square, i.e. $\left\{\left(k_{x}, k_{y}\right) \mid-\pi / a<\right.$ $\left.k_{x} \leq \pi / a,-\pi / a<k_{y} \leq \pi / a\right\}$. However, as a consequence of the time inversion symmetry, and of the mirror symmetry present in the perfect approximants (see Sec. 2), certain parts of the Brillouin zone give the same contribution to the density of states, so that at least for the perfect approximants the irreducible part over which the integration has to be carried out is only a quarter of the total Brillouin zone, i.e. $\left\{\left(k_{x}, k_{y}\right) \mid 0 \leq k_{x} \leq \pi / a,-k_{x} \leq k_{y} \leq k_{x}\right\}$.

For the numerical integration (4) over the branch surfaces, the irreducible part of the Brillouin zone is split into equal, right-angled isosceles triangles, called simplices. The density of the grid is indicated by an integer number $m$, which is equal to the number of times that the short edge length of a simplex fits into the interval $[0, \pi / a]$. The total number of simplices contained in the irreducible part of the Brillouin zone is then equal to $2 m^{2}$ ( $4 m^{2}$ in the case of randomized approximants). Within each simplex the branch surface is interpolated quadratically as:

$$
\omega_{s}(\mathbf{k})=a+\mathbf{b}^{T} \mathbf{k}+\mathbf{k}^{T} C \mathbf{k}
$$

where the six free parameters of the scalar $a$, the two component vector $b$ and the symmetric $(2 \times 2)$-matrix $C$ are fixed by prescribing the values at the corners and the mid-edges of the triangles, which are obtained by numerical diagonalisation of the dynamical matrix. Subsequently, each simplex is split up into smaller simplices of exactly the same form, and within each of these smaller simplices the branch surface is interpolated linearly:

$$
\omega_{s}(\mathbf{k})=a^{\prime}+\mathbf{b}^{\prime T} \mathbf{k},
$$

where the three free parameters of $a^{\prime}$ and $\mathbf{b}^{\prime}$ are fixed by the values at the corners of the small simplex, which are evaluated using the quadratic fit (5). This method is known as the hybrid method. With the linear expression (6) the integration (4) becomes quite easy. 
Using a computer, one usually determines the density of states $D(\omega)$ on a grid of frequencies $\omega_{i}$, separated by a step length $\Delta \omega$. The value of $D\left(\omega_{i}\right)$ is calculated by adding the contributions from all the simplices whose frequency support contains $\omega_{i}$. This implies that the contributions from a simplex gets lost whenever the frequency support of a simplex is entirely contained between two grid points. Such situations will occur in particular when the branches are very flat, which is indeed the case for the phonon branches of the OT-approximants (see Fig. 4). One may solve this difficulty by taking a very dense frequency grid, but this reduces the efficiency of the computation. Here we propose a different solution. To make sure that the contribution of each of the simplices is taken entirely into account, we determine the contribution from a simplex to $D\left(\omega_{i}\right)$ by evaluating the following integral:

$$
D\left(\omega_{i}\right)=\int_{\omega_{\text {mix }}}^{\omega_{\max }} d \omega \frac{F_{i}(\omega) L(\omega)}{\left|\hat{\mathbf{n}} \nabla \omega_{s}(\mathbf{k})\right|}
$$

where $\omega_{\min }$ and $\omega_{\max }$ are the minimum and the maximum of the frequency support of the linearly interpolated branch surface within the simplex, $L(\omega)$ is the length of the line segment of constant $\omega$ inside the simplex and the function $F_{i}(\omega)$ is a weight function. An obvious choice for $F_{i}(\omega)$ would be the function which has the value 1 on the interval $\left(\omega_{i}-\Delta \omega / 2, \omega_{i}+\Delta \omega / 2\right]$ and is 0 elsewhere. A slightly more sophisticated choice is the following:

$$
F_{i}(\omega)=\left\{\begin{array}{cl}
\frac{\Delta \omega-\left|\omega-\omega_{i}\right|}{\Delta \omega} & \omega \in\left[\omega_{i}-\Delta \omega, \omega_{i}+\Delta \omega\right] \\
0 & \text { elsewhere }
\end{array} .\right.
$$

With this choice a contribution which falls between two grid points on the frequency axis is equally shared by these two points. To facilitate the integration, one can change to another integration variable $t$, which is related to $\omega$ by:

$$
\omega(t)=\omega_{\min }+\left(\omega_{\max }-\omega_{\min }\right) t .
$$

Substitution of (9) into (7) leads to:

$$
D\left(\omega_{i}\right)=\frac{\left(\omega_{\max }-\omega_{\min }\right)}{\left|\hat{\mathbf{n}} \nabla \omega_{3}(\mathbf{k})\right|} \int_{0}^{1} d t F_{i}(\omega(t)) L(\omega(t))
$$

where we have used the fact that $\left|\hat{\mathbf{n}} \nabla \omega_{\mathbf{s}}(\mathbf{k})\right|$ is a constant for the linear fit of the branch surface. This linearity of the branch surface as a function of $\mathbf{k}$ is also the reason for the linearity of the dependence of the length $L(\omega(t))$ on $t$. Its derivative, however, contains in general one discontinuity on the interval $t \in(0,1)$, namely at the value of $t$ for which the line segment $L(t)$ passes the corner of the triangle for which the value of $\omega$ lies between $\omega_{\min }$ and $\omega_{\max }$. Note that the values at the two other corners of the simplex are, respectively, $\omega_{\min }$ and $\omega_{\max }$. The length of the line segment at this $t$-value fixes completely the linear function $L(t)$ on the interval $[0,1]$, so that for each simplex only one length has to be determined. 


\subsection{Presentation of the density of states}

The effectiveness of the integration method is clearly demonstrated in Fig. 2, where the DOS is presented for the square lattice (solid line). The two singularities that occur due to the transverse and the longitudinal branch are nicely displayed. The dotted line in Fig. 2 represents the result for the OT-approximant with 7 atoms inside the unit cell, and illustrates how the strong singularities occurring for the square lattice are replaced by a number of much weaker peaks. In both cases a grid 10 interpolation was used in the integration method.

Figure 3 shows the DOS for the perfect 41-approximant using a grid 20 interpolation (solid line) and a grid 10 interpolation (dotted line). The dotted line is hardly visible, which means that for the grid 10 interpolation the DOS has almost converged. In fact, this was to be expected, since the branches are very flat, especially in the high frequency region. This is illustrated in Fig. 4, where the branches are displayed along a path in the Brillouin zone.

The DOS of the perfect 239-approximant is shown in Fig. 5a, obtained using a grid 10 interpolation. Comparing this with the DOS of the 41-approximant (Fig. 2), one observes that global convergence is obtained already at this level of commensurate approximation. Even some of the singularities remain at the same place. The majority of the finer details still change, however, but it may be expected that also these details of the DOS-curve will converge rapidly for larger approximants.

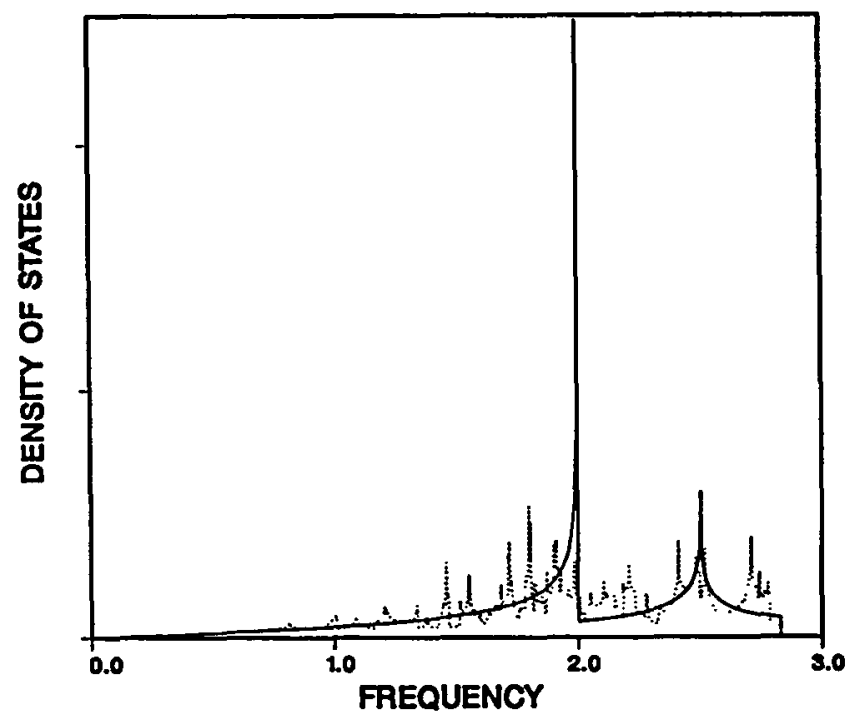

Fig. 2. The normalized density of states for the square lattice (solid line) and for the 7-approximant (dotted line) obtained using grid 10 interpolation. 


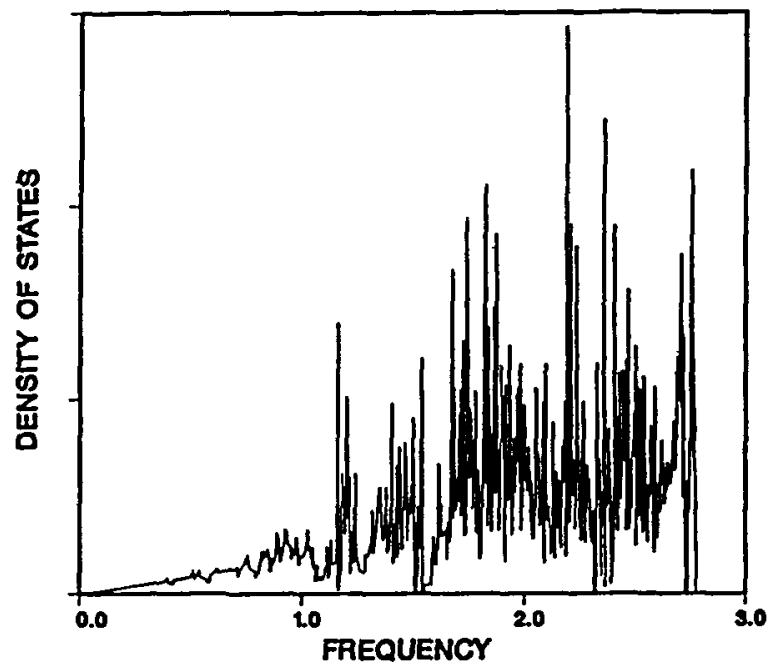

Fig. 3. The normalized density of states for the 41-approximant using grid 20 (solid line) and grid 10 interpolation (dotted line). Due to the good convergence, the dotted line can hardly be seen.

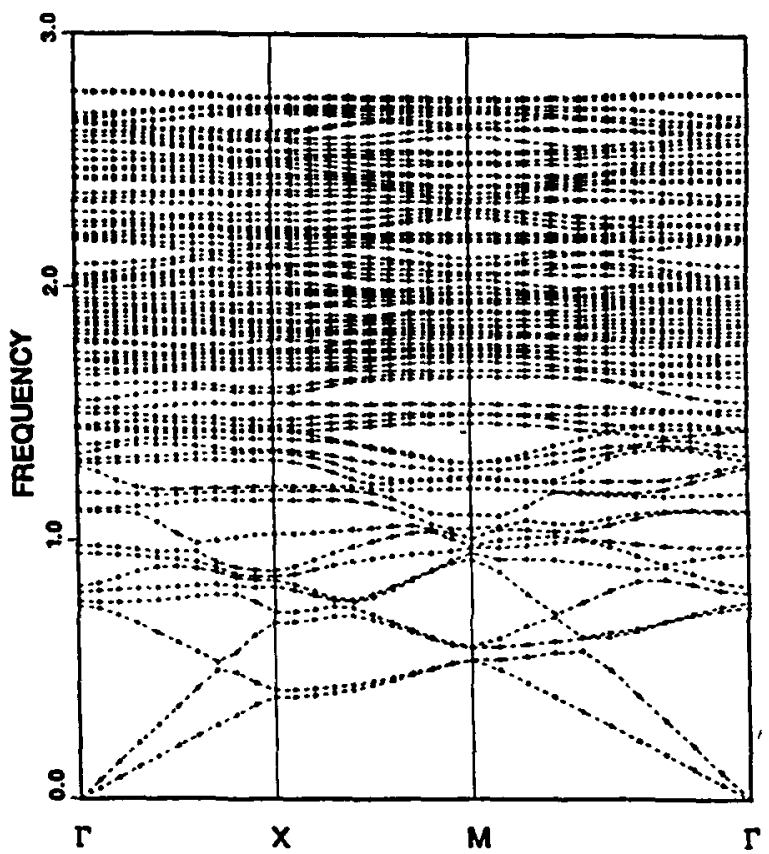

Fig. 4. The phonon branches along a path in the Brillouin zone for the 41-approximant. $\Gamma=0$, $X=\pi / a(1,0), M=\pi / a(1,1)$. 
The global contours of the DOS are destroyed by randomization, however, which is illustrated in Fig. 5b, where the DOS is presented for a randomized 239approximant. The same effect was also observed in three dimensions for the icosahedral Penrose tiling. ${ }^{4,5}$ Obviously, the special local order, which exists only in the perfect approximants, leads to the very specific behaviour of the DOS. That this local order indeed plays an important role for the global behaviour of the DOS is demonstrated by the fact that the DOS converges already for the lowest approximants. Note that the periodicity of the lower order approximants is still approximately present in the higher order approximants, and vice versa. For each of the perfect approximants (except for the 7-approximant), exactly the same finite set of neighbour configurations occurs in the structure. For the randomized approximants, however, additional neighbour configurations show up.

As an application, we have calculated the specific heat for different approximants, and compare it to the results for a square lattice and for a simple Debye model, where the DOS is assumed to be a linear function of $\omega$, cut off at some limiting frequency $\omega_{D}$. Recall that the mean energy of a quantum oscillator of frequency $\omega$ and at temperature $T$ is given by

$$
\langle E(\omega, T)\rangle=\frac{\hbar \omega}{2}+\frac{\hbar \omega}{\exp \left(\frac{\hbar \omega}{k_{B} T}\right)-1}
$$

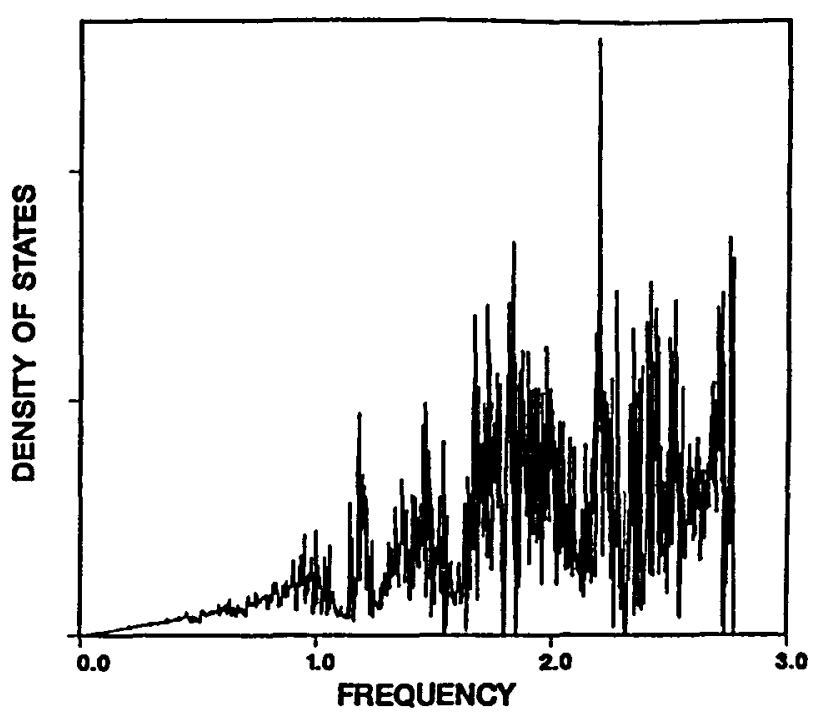

Fig. 5a. The normalized density of states for the perfect 239-approximant using grid 10 interpolation. 


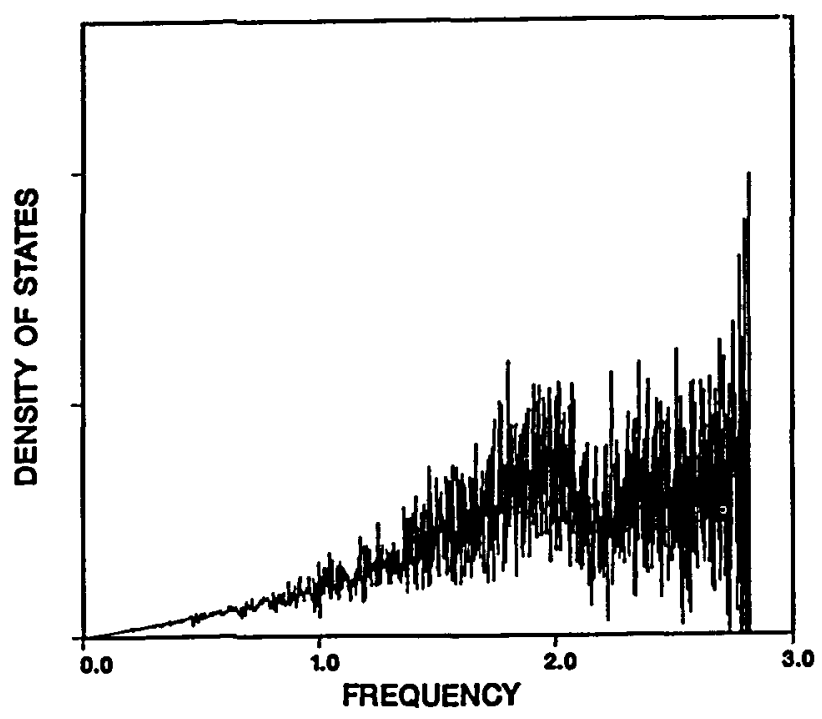

Fig. 5b. The normalized density of states for a randomized 239-approximant using grid 10 interpolation.

If $D(\omega)$ is the normalized density of states of an ensemble of oscillators, the inner energy per mode of this ensemble is given by

$$
U(T)=\int D(\omega)(E(\omega, T)\rangle d \omega,
$$

so that the specific heat $C(T)=\frac{\partial U(T)}{\partial T}$ becomes:

$$
C(T)=k_{B} \int D(\omega) \frac{\left(\frac{\hbar \omega}{k_{B} T}\right)^{2} \exp \left(\frac{\hbar \omega}{k_{B} T}\right)}{\left(\exp \left(\frac{\hbar \omega}{k_{B} T}\right)-1\right)^{2}} d \omega
$$

In Fig. 6, the specific heat curves are shown for a perfect and a randomized 1393approximant. These two curves almost exactly agree with each other, indicating that the specific heat is not very sensitive to the details of the DOS. In the same figure, the specific heat for the corresponding model on a square lattice is plotted, as well as the specific heat of a Debye model, with suitably fitted Debye temperature $\theta_{D}=\hbar \omega_{D} / k_{B}$. Note that the frequency or temperature scales for the OTapproximants and the square lattice are directly comparable, because the spring constants have been chosen such that the same average spring constant per atom was obtained in all three cases, whereas in the Debye case the cutoff frequency $\omega_{D}$ (and thus the Debye temperature $\theta_{D}$ ) had to be suitably adjusted to make the curves 


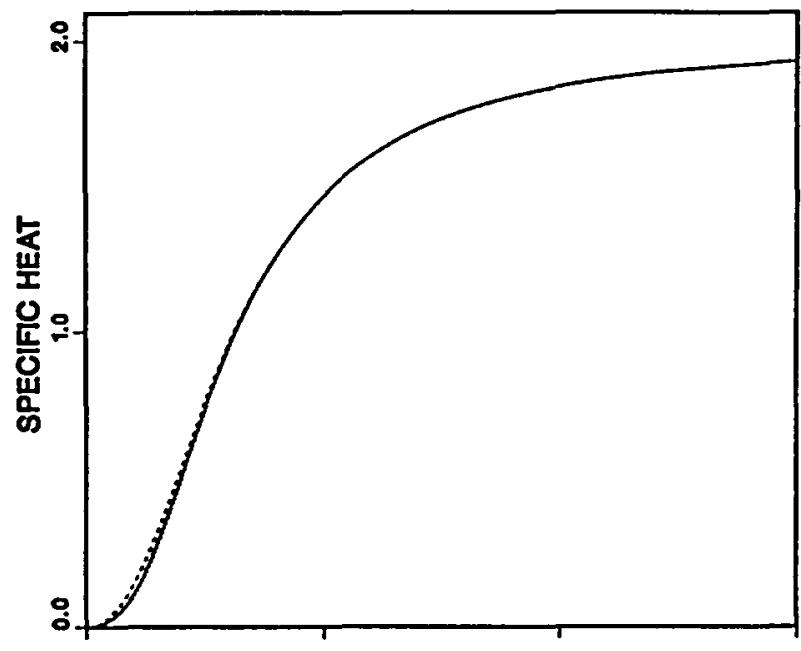

TEMPERATURE

Fig. 6. The vibrational specific heat per atom for the different models, in units of $k_{B}$. The scale on the temperature axis is arbitrary. Shown are the specific heats of a perfect and a randomized 1393-approximant (solid line and chaindashed line lying on top of one another), a square lattice (dotted line) and a Debye model (dashed line).

comparable. At low temperatures, the square lattice specific heat deviates slightly from that of the OT-approximants, but this is only a minor deviation. Somewhat more important is the deviation of the Debye specific heat at low temperatures. Note that we have used a Debye temperature for which an overall good fit is obtained. If, instead, the Debye temperature would be chosen such that the $T^{2}$-terms at low temperature would agree, then the Debye specific heat would be substantially reduced at higher temperature, with respect to the other curves. Apparently, the simple Debye model is not very good in two dimensions, although the differences are not very important. A similar calculation for 3-dimensional icosahedral Penrose tilings yielded, however, a much better fit between quasiperiodic, periodic and Debye models. Nevertheless, Fig. 6 shows that it is qualitatively correct to use a Debye model for the vibrational specific heat, as it has been done in Ref. 19.

In the low frequency limit the DOS is linear in $\omega$, as a consequence of the linear dispersion of the acoustic branches. However, beyond the frequency support of the acoustic branches, gaps will occur in the dispersion, which lead to pseudogaps in the DOS. Figure 7 shows the low frequency part of the DOS for the perfect 239approximant, using a grid 20 interpolation (solid line). The dotted line represents the result using a grid 10 interpolation, illustrating that also on this scale all significant features are already present for the lower grid density. Figure $8 \mathrm{a}$ shows the DOS for the 1393-approximant on the same frequency interval, and Fig. $8 \mathrm{~b}$ presents 


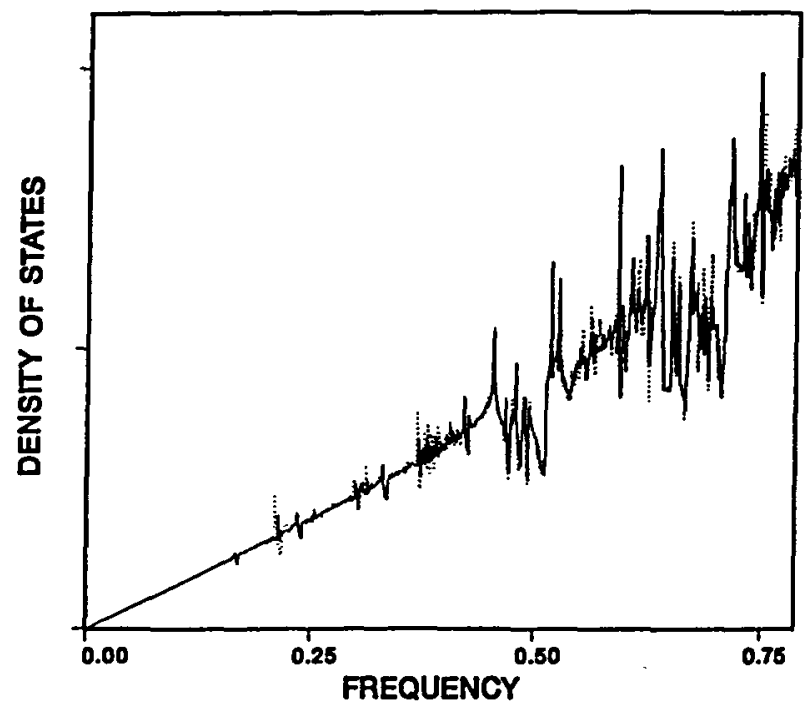

Fig. 7. The density of states at low frequencies for the perfect 239-approximant using grid 20 (solid line) and grid 10 interpolation (dotted line).

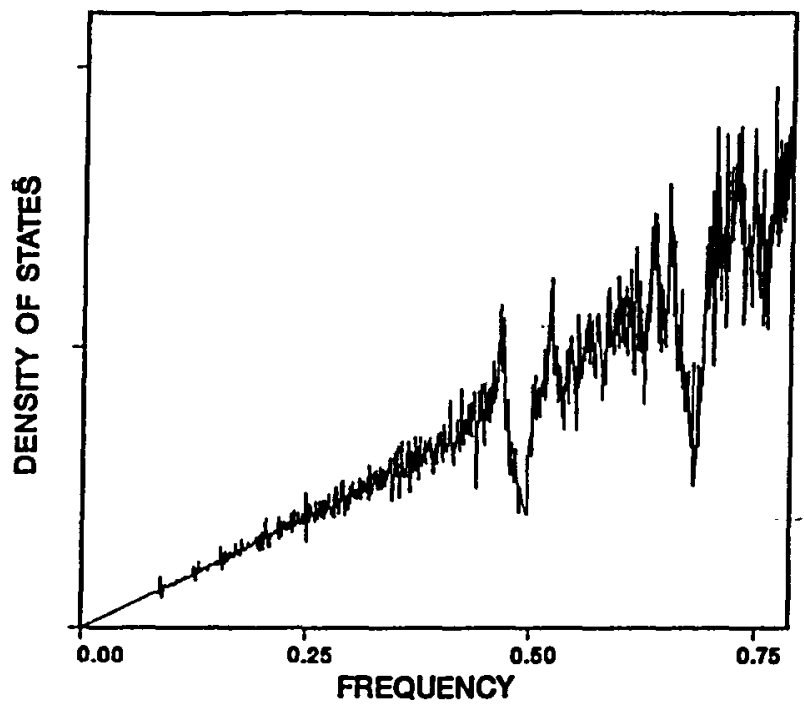

Fig. 8s. The density of states at low frequencies for the perfect 1393-approximant using grid 10 interpolation. 


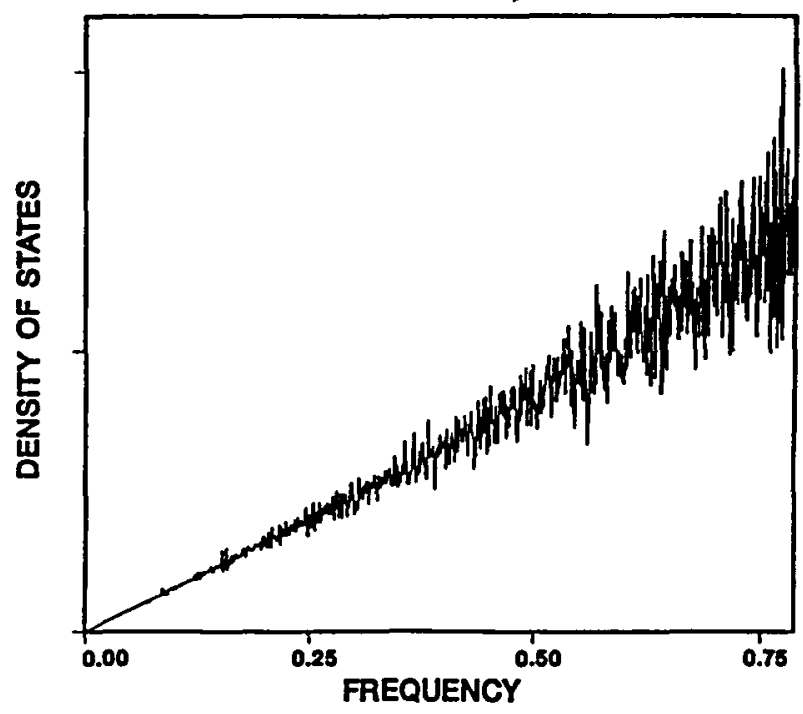

Fig. 8b. The density of states at low frequencies for a randomized 1393-approximant using grid 10 interpolation.

the same result for a randomized approximant, both obtained using a grid $10 \mathrm{in}$ terpolation. The pseudogap occurring at about 0.5 for the perfect approximant is also present in the DOS for the 41-approximant (Fig. 3) and the 239-approximant (Fig. 7), suggesting that the situation has essentially converged and will not change significantly any more as the quasiperiodic limit is approached. Below $\omega=0.5$ no significant new gaps occur in the DOS when higher approximants are considered. The effect of randomization is the same as was observed for the 239-approximant, namely that the pseudogaps disappear.

\section{Scaling Behaviour of the Phonon Branches}

The character of the spectrum in the incommensurate limit may be obtained by analysing the decay of the bandwidths for successive rational approximants. This was also done for quasiperiodic systems in one dimension by Kohmoto, ${ }^{13}$ who introduced a method to derive the character of the spectrum, i.e., either absolutely continuous, singularly continuous or pure point, from the convergence of the socalled spectral $S(\epsilon)$-function. In Kohmoto's method the $S(\epsilon)$-function is calculated using a thermodynamical formalism.

The method can be generalized to two and three dimensions as follows. A measure for the size of the system is defined by:

$$
L=(s N)^{(1 / s)}
$$


where $s$ is the dimension of the system and $N$ the number of atoms inside the unit cell of the approximant, so that $s N$ is equal to the number of branches. For each band a scaling index $\epsilon_{i}$ is defined by:

$$
\Delta_{i}=(1 / L)^{\epsilon_{s}}
$$

where $\Delta_{i}$ is the bandwidth of the $i$ th band, which we will define later on for the OT-approximants. The set of scaling indices gives rise to a distribution $\Omega(\epsilon)$, which is defined such that $\Omega(\epsilon) d \epsilon$ is equal to the number of bands with scaling index between $\epsilon$ and $\epsilon+d \epsilon$. The spectral $S(\epsilon)$-function is related to $\Omega(\epsilon)$ by:

$$
S(\epsilon)=\frac{\ln (\Omega(\epsilon))}{\ln (L)}
$$

For certain 1-dimensional quasiperiodic systems, convergence of the $S(\epsilon)$-function was observed. ${ }^{20}$

Instead of considering the $S(\epsilon)$, and using the thermodynamical formalism, ${ }^{13}$ which often leads to numerical difficulties, we introduce a distribution $T(\epsilon)$, which is derived as follows. First, we switch to the normalized distribution $\hat{\Omega}(\epsilon)$, which is equal to $\Omega(\epsilon) / s N$, so that $\int_{\epsilon_{\min }}^{\epsilon_{\text {max }}} \hat{\Omega}(\epsilon) d \epsilon=1$. Substituting this into (16) gives:

$$
S(\epsilon)-s=\frac{\ln (\hat{\Omega}(\epsilon))}{\ln (L)} .
$$

Then, define a distribution $T(\epsilon)$ by:

$$
T(\epsilon)=e^{S(\epsilon)-s}=\hat{\Omega}(\epsilon)^{1 / \ln (L)} .
$$

This definition implies that if $S(\epsilon)$ converges, then $T(\epsilon)$ converges too. A slightly smoothened and continuous distribution $\Omega(\epsilon)$ can be obtained by using:

$$
\Omega(\epsilon)=\frac{1}{\pi} \operatorname{Im} \sum_{i} \frac{1}{\epsilon-\epsilon_{i}-i \nu}
$$

where $\nu$ a small positive number, which governs the rate of smoothening. Determination of $T(c)$ is then straightforward.

Besides the numerical advantages of considering $T(\epsilon)$ instead of $S(\epsilon)$, there is also the advantage that $T(\epsilon)$ contains more details about the distribution of scaling indices. However, in the limit of large systems both functions provide the same information concerning the characterization of the spectrum. If all bandwidths scale proportional to $1 / L$, then the spectrum is absolutely continuous, and the support of the $T(\epsilon)$-function is just one point, i.e. $\epsilon=1$ and $T(\epsilon)=1$. For a pure point spectrum, the bandwidths decay exponentially in $L$, and all scaling indices tend to infinity, so that $S(\infty)=s$. In the intermediate case, the support of the 
$T(\epsilon)$-function is some finite interval, which corresponds to a singularly continuous spectrum.

To study the scaling behaviour of the branches of the OT-approximants, the following definition of bandwidth is used:

$$
\Delta_{i}=\max _{j, j^{\prime}=1,2,3}\left|\omega_{i}\left(\mathbf{k}_{j}\right)-\omega_{i}\left(\mathbf{k}_{j^{\prime}}\right)\right|
$$

where $i$ labels the branches and $k_{1}, k_{2}$ and $k_{3}$ are respectively the wavevectors 0 , $\frac{\pi}{a}(1,0)$ and $\frac{\pi}{a}(1,1)$, with $a$ the lattice constant of the approximant. This definition is in general not the true bandwidth, but it can nevertheless be used to study the scaling behaviour of the bands.

The $T(\epsilon)$-functions for the 41-, 239-and 1393-approximants are shown in Fig. 9a. In each of the three cases, the lower and the upper bound of the support of the $T(\epsilon)$ function was determined by, respectively, the minimal and the maximal scaling index occurring in the set. The distribution $\Omega(\epsilon)$ then was normalized over this interval. To illustrate the difference relative to the behaviour for an absolutely continuous spectrum, Fig. 9b shows the $T(\epsilon)$-functions for a square lattice, taking a unit cell with 100 atoms (dotted line), and one with 400 atoms. The bandwith was defined in the same way as for the OT-approximants, labelling the eigenfrequencies at the three $k$-vectors in ascending order. Although the picture demonstrates that convergence to $\epsilon=1$ can be very slow, the averaged scaling index is significantly lower than for the OT-approximants. Note that a difference of 1 for the scaling index means a difference of a factor $1 / L$ for the bandwidths. For the OT-approximants the distribution has a peak at about $\epsilon=2$ in all three case, which makes it unlikely that the scaling indices will converge to 1 in the quasiperiodic limit. Therefore we conjecture that the phonon spectrum of the octagonal tiling has to be classified as singularly continuous.

However, Fig. 9a also shows that the lowest scaling indices, which correspond to the accoustic branches, are approaching the value 1 when tending to higher approximants. To have a closer look at the scaling behaviour of the lowest branches, Figs. 10a, 10b and 10c show the lowest 18 branches along a path inside the Brillouin zone for, respectively, the perfect 239-, 1393-, and 8119-approximants. Comparing the 239- and the 1393-approximant, we observe that the width of the gaps relative to the bandwidth has decreased for the 1393-approximant. Let us look, for example, at the gap near $\omega=0.48$, which occurs for the 239-approximant at the $X$-point. This gap scales to the relatively much smaller gap at about $\omega=0.2$ for the 1393approximant. For the 8119 this trend persists. Therefore we may expect that the scaling behaviour of the lowest branches is exact in the incommensurate limit, i.e. the band patterns will become exact copies of one another, only scaled by a factor $1+\sqrt{2}$ on the frequency axis. In fact, this band pattern then is just the reduced scheme of a normal linear dispersion in each direction. For the $T(\epsilon)$-function this means that the lower bound of the support will converge to 1 in the incommensurate limit. 


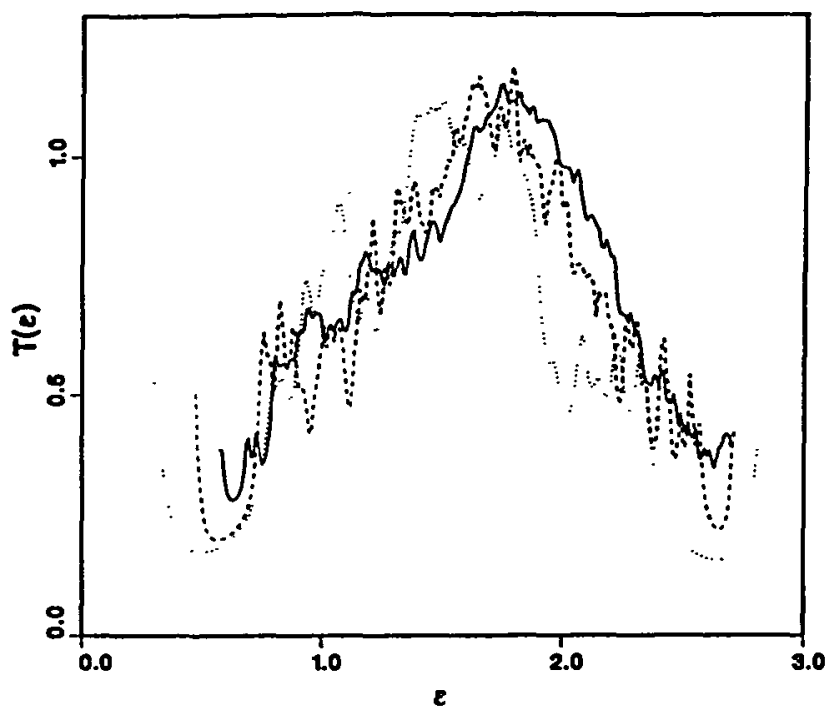

Fig. 9a. The spectral $T(\epsilon)$-function for the perfect 41-, 239- and 1393-approximant (respectively dotted, dashed and solid line).

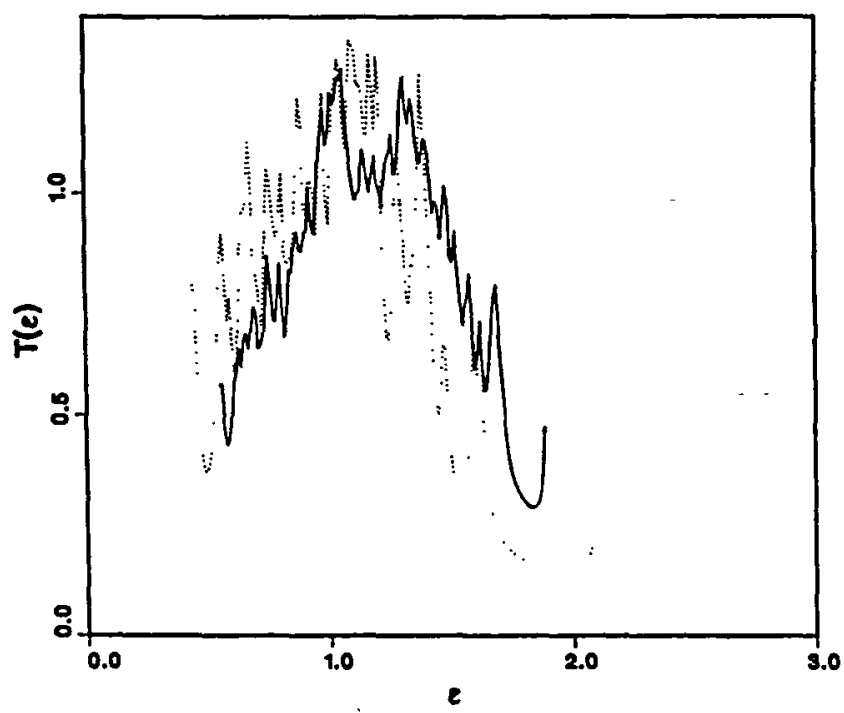

Fig. 9b. The spectral $T(c)$-function for the square lattice using a unit cell with 100 atoms (dotted line) and one with 400 atoms (solid line). 


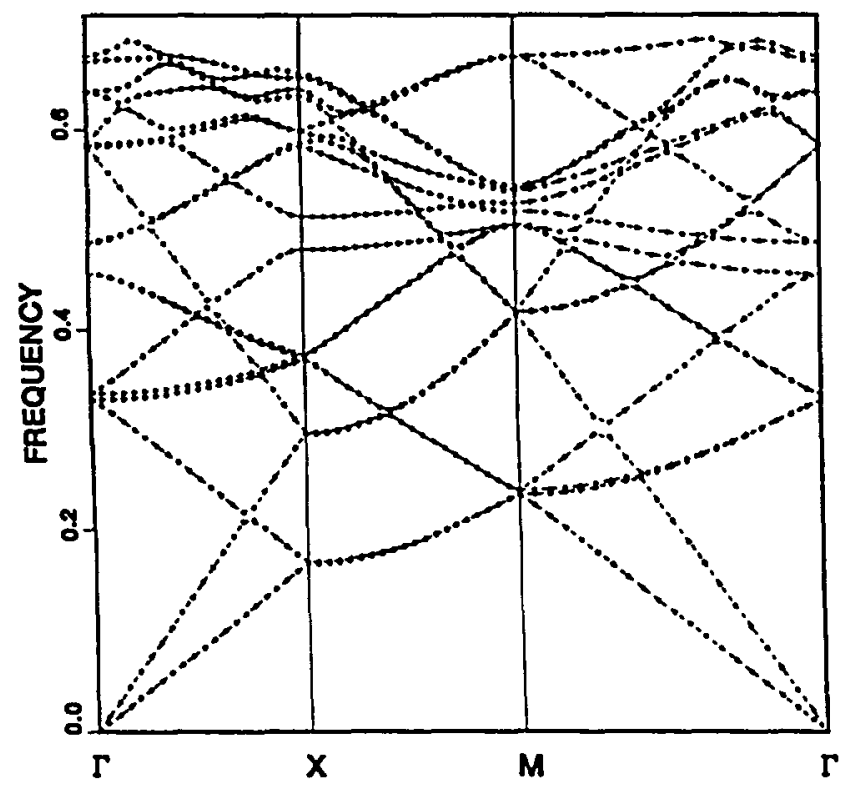

Fig. 10a. The lowest 18 branches along a path in the Brillouin zone for the perfect 239-approximant (see also Fig. 4).



Fig. 10b. Same as in Fig. 9a, but for the perfect 1393-approximant. 


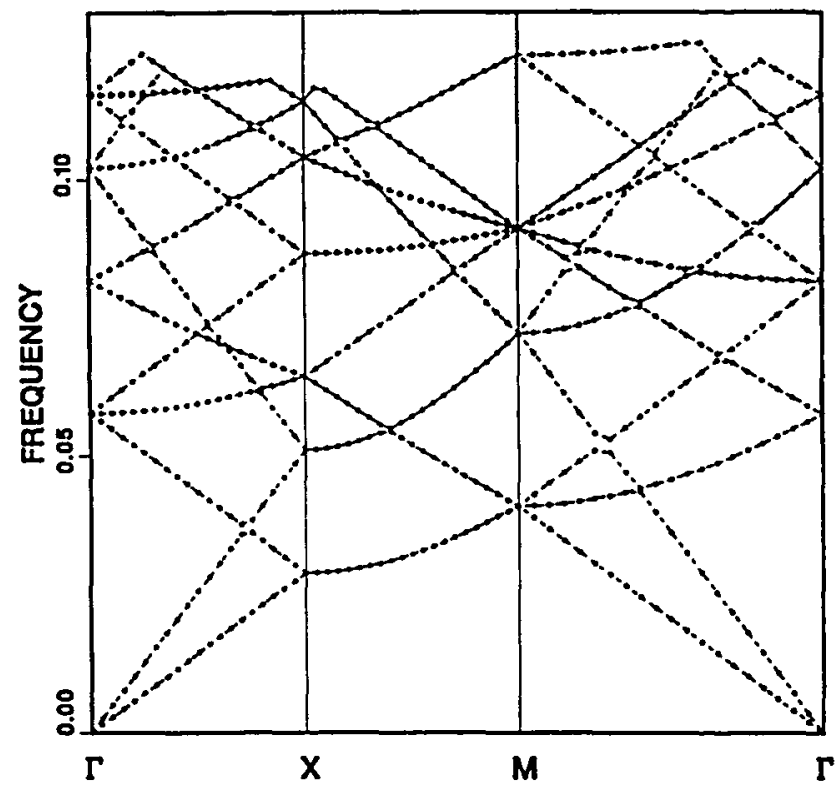

Fig. 10c. Same as in Fig. 9a, but for the perfect 8119-approximant.

\section{Concluding Remarks}

From our studies of the vibrational spectra of perfect and randomized approximants of the octagonal tiling the following conclusions on the vibrational spectra of the octagonal tiling itself can be drawn.

The vibrational density of states of the octagonal tiling exhibits much structure, with a rich pattern of pseudogaps, and probably also true gaps at high frequencies. The density of states is considerably affected by randomization. Randomization induces a global smoothening, even at relatively low frequencies, and pseudogaps mostly disappear.

According to the scaling behaviour of the bandwidths for the rational approximants, the spectrum of the octagonal tiling has to be classified as singularly continuous. However, in the low frequency limit the scaling behaviour corresponds to an absolutely continuous behaviour. Qualitatively this scaling behaviour is similar to that of the phonon spectra for 1-dimensional quasi-crystals.

These results agree with what has been obtained in previous studies ${ }^{8,9}$ on the phonon spectra of 2-dimensional quasiperiodic systems. Interestingly, similar scaling properties of the spectrum have been found also for electronic systems based on the octagonal tiling. ${ }^{21,22}$ 


\section{Acknowledgments}

Our randomization program is partly based on the code written by L.H. Tang. We thank him for freely sharing his code with us. This work has been supported by the 'Stichting voor Fundamenteel Onderzoek der Materie' (F.O.M.) with financial support of the 'Nederlandse Organisatie voor Wetenschappelijk Onderzoek' (N.W.O.).

\section{References}

1. G. Gumbs and M. K. Ali, Phys. Rev. Lett. 60, 1081 (1988).

2. J. M. Luck and D. Petritis, J. Stat. Phys. 42, 289 (1986).

3. J. Los and T. Janssen, J. Phys.: Condens. Matter 2, 9553 (1990).

4. J. Los, T. Janssen and F. Gähler, "Lattice dynamics of 3-dimensional tilings modelling icosahedral quasicrystals", to appear in J. Non-Cryst. Solids.

5. J. Los, T. Janssen and F. Gähler, J. Phys. I (France) 3, 107 (1993).

6. G. Kasner and H. Böttger, to appear in J. Non-Cryst. Solids.

7. G. Kasner and H. Böttger, this volume.

8. J. A. Ashraff, J.-M. Luck and R. B. Stinchcombe, Phys. Rev. B41, 4314 (1990).

9. Z. Liu, Z. Zhang, Q. Jiang, and D. Tian, J. Phys.: Condens. Matter 4, 6343 (1992).

10. A. H. MacDonald, S. H. Vosko and P. T. Coleridge, J. Phys.: Condens. Matter 12, L949 (1979).

11. L. H. Methfessel, J. Phys. Condens. Matter 16, 949 (1983).

12. M. Kohmoto and J. R. Banavar, Phys. Rev. B34, 563 (1986).

13. M. Kohmoto, Phys. Rev. A37, 135 (1988).

14. B. Grūnbaum and G.C. Shephard, Tilings and Patterns (Freeman, New York, 1986).

15. F. P. M. Beenker, Eindhoven University of Technology Technical Report No. 82-WSK04 (1982).

16. F. Gāhler and J. Rhyner, J. Phys. A: Math. Gen. 19, 267 (1986).

17. C. L. Henley, "Random Tiling Models", in Quasicrystals: The State of the Art, ed. P. J. Steinhardt and D. P. DiVincenco (World Scientific, 1991).

18. L. H. Tang, Phys. Rev. Lett. 64, 2390 (1990).

19. M. Baake and D. Joseph, Phys. Rev. B42, 8091 (1990).

20. T. Janssen and M. Kohmoto, Phys. Rev. B38, 5811 (1988).

21. C. Sire, Europhys. Lett. 10, 483 (1989).

22. C. Sire and J. Belissard, Europhys. Lett. 11, 439 (1990). 